\title{
BIBECHANA
}

A Multidisciplinary Journal of Science, Technology and Mathematics ISSN 2091-0762 (Print), 2382-5340 (Online) Journal homepage: http://nepjol.info/index.php/BIBECHANA

Publisher: Research Council of Science and Technology, Biratnagar, Nepal

\section{Generalizations of some Enestrom-Kakeya type results}

\section{M.H. Gulzar*, A.W. Manzoor}

Department of Mathematics, University of Kashmir, Srinager-190006, Jammu and Kashmir, India.

"E-mail: gulzarmh@gmail.com

Article history: Received 05 June, 2015; Accepted 20 August, 2015

DOI: http://dx.doi.org/10.3126/bibechana.v13i0.13309

\section{Abstract}

In this paper we give interesting generalizations of some well-known Enestrom-Kakeya type results on the location of zeros of a complex polynomial under less restrictive conditions on the coefficients of the polynomial.

CRCOST: All rights reserved.

Keywords: Bound; Coefficient; Polynomial; Zeros.

\section{Introduction}

Regarding the zeros of a polynomial with real and positive coefficients, we have the following result known as the Enestrom-Kakeya Theorem [1, 2, 3].

Theorem A: Let $P(z)=\sum_{j=0}^{n} a_{j} z^{j}$ be a polynomial of degree $n$ such that

$$
a_{n} \geq a_{n-1} \geq \ldots . . \geq a_{1} \geq a_{0}>0 .
$$

Then all the zeros of $P(z)$ lie in $|z| \leq 1$.

Various extensions and generalizations of this result are available in the literature. Joyal et al [4] proved the following generalization of Theorem A:

Theorem B: Let $P(z)=\sum_{j=0}^{n} a_{j} z^{j}$ be a polynomial of degree $n$ such that

$$
a_{n} \geq a_{n-1} \geq \ldots . . . \geq a_{1} \geq a_{0} .
$$

Then $P(z)$ has all its zeros in the disk

$$
|z| \leq \frac{a_{n}-a_{0}+\left|a_{0}\right|}{\left|a_{n}\right|} .
$$

As a generalization of Theorems A and B, Aziz and Zargar [5] proved the following:

Theorem C: Let $P(z)=\sum_{j=0}^{n} a_{j} z^{j}$ be a polynomial of degree $n$ such that for some $k \geq 1$

$$
k a_{n} \geq a_{n-1} \geq \ldots . . \geq a_{1} \geq a_{0} .
$$


Then $P(z)$ has all its zeros in the disk

$$
|z+k-1| \leq \frac{k a_{n}-a_{0}+\left|a_{0}\right|}{\left|a_{n}\right|} .
$$

Shah et al [6] extended Theorem B to polynomials with complex coefficients and proved the following result:

Theorem D: Let $P(z)=\sum_{j=0}^{n} a_{j} z^{j}$ be a complex polynomial of degree $n$ with $\operatorname{Re}\left(a_{j}\right)=\alpha_{j}$ and $\operatorname{Im}\left(a_{j}\right)=\beta_{j}$ for $j=0,1,2, \ldots \ldots, n$, such that for some $k \geq 1$

$$
\begin{aligned}
& k \alpha_{n} \geq \alpha_{n-1} \geq \ldots . . \geq \alpha_{1} \geq \alpha_{0} \\
& \beta_{n} \geq \beta_{n-1} \geq \ldots . . \geq \beta_{1} \geq \beta_{0}>0 .
\end{aligned}
$$

Then $P(z)$ has all its zeros in the disk

$$
\left|z+\frac{\alpha_{n}}{a_{n}}(k-1)\right| \leq \frac{k \alpha_{n}-\alpha_{0}+\alpha_{0} \mid+\beta_{n}}{\left|a_{n}\right|} .
$$

Liman et al [7] proved the following generalization of Theorem D:

Theorem E: Let $P(z)=\sum_{j=0}^{n} a_{j} z^{j}$ be a complex polynomial of degree $n$ with $\operatorname{Re}\left(a_{j}\right)=\alpha_{j}$ and $\operatorname{Im}\left(a_{j}\right)=\beta_{j}$ for $j=0,1,2, \ldots \ldots, n, a_{n} \neq 0$. If for some positive integer $\lambda \leq n$ and $k \geq 1$

$$
\begin{gathered}
k^{n-\lambda+1} \alpha_{n} \geq k^{n-\lambda} \alpha_{n-1} \geq k^{n-\lambda-1} \alpha_{n-2} \geq \ldots \ldots \geq k^{2} \alpha_{\lambda+1} \geq k \alpha_{\lambda} \geq \alpha_{\lambda-1} \geq \ldots \ldots \geq \alpha_{1} \geq \alpha_{0}, \\
\beta_{n} \geq \beta_{n-1} \geq \ldots \ldots \geq \beta_{1} \geq \beta_{0}>0,
\end{gathered}
$$

then all the zeros of $P(z)$ lie in

$$
\left|z+\frac{\alpha_{n}}{a_{n}}(k-1)\right| \leq \frac{\alpha_{n}-\alpha_{0}+\left|\alpha_{0}\right|+(k-1)\left(\sum_{j=\lambda}^{n}\left(\alpha_{j}+\left|\alpha_{j}\right|\right)-\left|\alpha_{n}\right|\right)+\beta_{n}}{\left|a_{n}\right|} .
$$

In the same paper they proved the following result also.

Theorem F: Let $P(z)=\sum_{j=0}^{n} a_{j} z^{j}$ be a complex polynomial of degree $n$ with $\operatorname{Re}\left(a_{j}\right)=\alpha_{j}$ and $\operatorname{Im}\left(a_{j}\right)=\beta_{j}$ for $j=0,1,2, \ldots \ldots, n, a_{n} \neq 0$. If for some positive integer $\lambda \leq n, k \geq 1$ and $\mu \geq 1$,

$$
\begin{gathered}
k^{n-\lambda+1} \alpha_{n} \geq k^{n-\lambda} \alpha_{n-1} \geq k^{n-\lambda-1} \alpha_{n-2} \geq \ldots \ldots \geq k^{2} \alpha_{\lambda+1} \geq k \alpha_{\lambda} \geq \alpha_{\lambda-1} \geq \ldots \ldots \geq \alpha_{1} \geq \alpha_{0}, \\
\mu \beta_{n} \geq \beta_{n-1} \geq \ldots \ldots . \beta_{1} \geq \beta_{0}>0
\end{gathered}
$$

then all the zeros of $P(z)$ lie in

$$
\left|z+\frac{k \alpha_{n}+i \mu \beta_{n}}{a_{n}}-1\right| \leq \frac{\alpha_{n}-\alpha_{0}+\left|\alpha_{0}\right|+(k-1)\left(\sum_{j=\lambda}^{n}\left(\alpha_{j}+\left|\alpha_{j}\right|\right)-\left|\alpha_{n}\right|\right)+\mu \beta_{n}}{\left|a_{n}\right|} .
$$

Recently Gulshan Singh [8] proved the following generalization of Theorems E and F: 
M. H. Gulzar and A. W. Manzoor/ BIBECHANA 13 (2016) 1-8 : RCOST p.3 (Online Publication: Dec., 2015)

Theorem G: Let $P(z)=\sum_{j=0}^{n} a_{j} z^{j}$ be a complex polynomial of degree $n$ with $a_{j}=\alpha_{j}+i \beta_{j}, j=0,1,2, \ldots \ldots, n$, where $\alpha_{j}$ and $\beta_{j}$ are real numbers. If for some positive integers $\lambda, \mu \leq n$ and for some real numbers $0<\rho_{1} \leq 1,0<\rho_{2} \leq 1, k \geq 1$, $k^{n-\lambda+1} \alpha_{n} \geq k^{n-\lambda} \alpha_{n-1} \geq k^{n-\lambda-1} \alpha_{n-2} \geq \ldots . . \geq k^{2} \alpha_{\lambda+1} \geq k \alpha_{\lambda} \geq \alpha_{\lambda-1} \geq \ldots \ldots \geq \alpha_{1} \geq \rho_{1} \alpha_{0}$, $k^{n-\mu+1} \beta_{n} \geq k^{n-\mu} \beta_{n-1} \geq k^{n-\mu-1} \beta_{n-2} \geq \ldots . . \geq k^{2} \beta_{\mu+1} \geq k \beta_{\mu} \geq \beta_{\mu-1} \geq \ldots \ldots \geq \beta_{1} \geq \rho_{2} \beta_{0}$, then all the zeros of $P(z)$ lie in

$$
\begin{gathered}
|z+k-1| \leq \frac{1}{\left|a_{n}\right|}\left[\left(\alpha_{n}+\beta_{n}\right)-\left(\rho_{1} \alpha_{0}+\rho_{2} \beta_{0}\right)+\left(1-\rho_{1}\right)\left|\alpha_{0}\right|+\left(1-\rho_{2}\right)\left|\alpha_{0}\right|+\left(\left|\alpha_{0}\right|+\left|\beta_{0}\right|\right)\right. \\
\left.+(k-1) \sum_{j=\lambda}^{n}\left(\alpha_{j}+\left|\alpha_{j}\right|\right)+\sum_{j=\mu}^{n}\left(\beta_{j}+\left|\beta_{j}\right|\right)-\left|\alpha_{n}\right|-|\beta|\right]^{n} .
\end{gathered}
$$

In this paper we prove the following result which not only generalizes the above results but also gives many other results for different values of the parameters.

\section{Theorems and Proofs}

Theorem 1. Let $P(z)=\sum_{j=0}^{n} a_{j} z^{j}$ be a complex polynomial of degree $n$ with $a_{j}=\alpha_{j}+i \beta_{j}, j=0,1,2, \ldots \ldots, n$, where $\alpha_{j}$ and $\beta_{j}$ are real numbers. If for some positive integers $\lambda, \mu \leq n$ and for some real numbers $0<\rho_{1} \leq 1,0<\rho_{2} \leq 1, k_{1} \geq 1, k_{2} \geq 1$,

$$
\begin{aligned}
& k_{1}{ }^{n-\lambda+1} \alpha_{n} \geq k_{1}{ }^{n-\lambda} \alpha_{n-1} \geq k_{1}{ }^{n-\lambda-1} \alpha_{n-2} \geq \ldots . . \geq k_{1}{ }^{2} \alpha_{\lambda+1} \geq k_{1} \alpha_{\lambda} \geq \alpha_{\lambda-1} \geq \ldots \ldots \geq \alpha_{1} \geq \rho_{1} \alpha_{0}, \\
& k_{2}{ }^{n-\mu+1} \beta_{n} \geq k_{2}{ }^{n-\mu} b \beta_{n-1} \geq k_{2}{ }^{n-\mu-1} \beta_{n-2} \geq \ldots . . \geq k_{2}{ }^{2} \beta_{\mu+1} \geq k_{2} \beta_{\mu} \geq \beta_{\mu-1} \geq \ldots . . \geq \beta_{1} \geq \rho_{2} \beta_{0},
\end{aligned}
$$

then all the zeros of $P(z)$ lie in

$$
\begin{aligned}
\mid z+\frac{\left(k_{1}-1\right) \alpha_{n}+i\left(k_{2}-1\right) \beta_{n} \mid \leq \frac{1}{a_{n} \mid}[}{\left|a_{n}\right|} & \left.\alpha_{n}+\beta_{n}\right)-\left(\rho_{1} \alpha_{0}+\rho_{2} \beta_{0}\right)+\left(1-\rho_{1}\right)\left|\alpha_{0}\right| \\
& +\left(1-\rho_{2}\right)\left|\beta_{0}\right|+\left(\left|\alpha_{0}\right|+\left|\beta_{0}\right|\right) \\
& +(k-1)\left(\sum_{j=\lambda}^{n}\left(\alpha_{j}+\left|\alpha_{j}\right|\right)\right)-\left(k_{1}-1\right)\left|\alpha_{n}\right| \\
& +\left(k_{2}-1\right)\left(\sum_{j=\mu}^{n}\left(\beta_{j}+\left|\beta_{j}\right|\right)-\left(k_{2}-1\right)\left|\beta_{n}\right|\right] .
\end{aligned}
$$

Taking $k_{1}=k_{2}=k, \rho_{1}=\rho_{2}=\rho$, in Theorem 1 we get Theorem G. Taking $k_{1}=k, k_{2}=1, \rho_{1}=\rho_{2}=1$, and $\beta_{0} \geq 0$ in Theorem 1 , we get Theorem E. Taking $k_{1}=k, k_{2}=\mu, \mu=n, \rho_{1}=\rho_{2}=1$ and $\beta_{0} \geq 0$ in Theorem 1 we get Theorem $\mathrm{F}$ of Liman et al.Taking $\lambda=n=\mu$ in Theorem 1, we get a Theorem of Gulzar [9,Theorem 1].

Taking $k_{1}=k_{2}=1=\rho_{1}=\rho_{2}$ in Theorem 1 we get the following interesting result: 
M. H. Gulzar and A. W. Manzoor/ BIBECHANA 13 (2016) 1-8 : RCOST p.4 (Online Publication: Dec., 2015)

Corollary 1. Let $P(z)=\sum_{j=0}^{n} a_{j} z^{j}$ be a complex polynomial of degree $n$ with $\operatorname{Re}\left(a_{j}\right)=\alpha_{j} \cdot \operatorname{Im}\left(a_{j}\right)=\beta_{j}$ for $j=0,1,2, \ldots \ldots, n$, such that

$$
\begin{aligned}
& \alpha_{n} \geq \alpha_{n-1} \geq \ldots . . \geq \alpha_{1} \geq \alpha_{0}, \\
& \beta_{n} \geq \beta_{n-1} \geq \ldots . . \geq \beta_{1} \geq \beta_{0} .
\end{aligned}
$$

Then $P(z)$ has all its zeros in the disk

$$
|z| \leq \frac{1}{\left|a_{n}\right|}\left(\alpha_{n}+\beta_{n}\right)
$$

Taking $a_{j}$ real, that is $\beta_{j}=0$ for $\mathrm{j}=0,1,2, \ldots \ldots, \mathrm{n}$ and $\alpha_{0}>0$,Corollary 1 reduces to Enestrom-Kakeya Theorem.

Taking $\lambda=\mu$ in Theorem 1, we get the following result:.

Corollary 2. Let $P(z)=\sum_{j=0}^{n} a_{j} z^{j}$ be a complex polynomial of degree $n$ with $\operatorname{Re}\left(a_{j}\right)=\alpha_{j} \cdot \operatorname{Im}\left(a_{j}\right)=\beta_{j}$ for $j=0,1,2, \ldots \ldots, n, a_{n} \neq 0$. If for some positive integer

$$
\begin{aligned}
\lambda \leq n, 0<\rho_{1} \leq 1,0 & <\rho_{2} \leq 1, k_{1} \geq 1, k_{2} \geq 1, \\
& k_{1}{ }^{n-\lambda+1} \alpha_{n} \geq k_{1}{ }^{n-\lambda} \alpha_{n-1} \geq k_{1}{ }^{n-\lambda-1} \alpha_{n-2} \geq \ldots . . \geq k_{1}{ }^{2} \alpha_{\lambda+1} \geq k_{1} \alpha_{\lambda} \geq \alpha_{\lambda-1} \geq \ldots \ldots . \alpha_{1} \geq \rho_{1} \alpha_{0}, \\
& k_{2}{ }^{n-\lambda+1} \beta_{n} \geq k_{2}{ }^{n-\lambda} \beta_{n-1} \geq k_{2}{ }^{n-\lambda-1} \beta_{n-2} \geq \ldots \ldots \geq k_{2}{ }^{2} \beta_{\lambda+1} \geq k_{2} \beta_{\lambda} \geq \beta_{\lambda-1} \geq \ldots \ldots \geq \beta_{1} \geq \rho_{2} \beta_{0},
\end{aligned}
$$

then all the zeros of $P(z)$ lie in

$$
\begin{aligned}
\mid z+\frac{\left(k_{1}-1\right) \alpha_{n}+i\left(k_{2}-1\right) \beta_{n} \mid \leq}{a_{n}} & \frac{1}{\left|a_{n}\right|}\left[\left(\alpha_{n}+\beta_{n}\right)-\left(\rho_{1} \alpha_{0}+\rho_{2} \beta_{0}\right)+\left(1-\rho_{1}\right)\left|\alpha_{0}\right|\right. \\
& +\left(1-\rho_{2}\right)\left|\beta_{0}\right|+\left(\left|\alpha_{0}\right|+\left|\beta_{0}\right|\right) \\
& +(k-1)\left(\sum_{j=\lambda}^{n}\left(\alpha_{j}+\left|\alpha_{j}\right|\right)\right)-\left(k_{1}-1\right)\left|\alpha_{n}\right| \\
& +\left(k_{2}-1\right)\left(\sum_{j=\lambda}^{n}\left(\beta_{j}+\left|\beta_{j}\right|\right)-\left(k_{2}-1\right)\left|\beta_{n}\right|\right] .
\end{aligned}
$$

Many other results may be deduced from Theorem 1 for different values of parameters.

Proof of Theorem 1: Consider the polynomial 


$$
\begin{aligned}
& F(z)=(1-z) p(z) \\
& =(1-z)\left(a_{n} z^{n}+a_{n-1} z^{n-1}+\ldots \ldots \ldots . .+a_{1} z+a_{0}\right) \\
& =-a_{n} z^{n+1}+\left(a_{n}-a_{n-1}\right) z^{n}+\ldots \ldots \ldots+\left(a_{1}-a_{0}\right) z+a_{0} \\
& =-a_{n} z^{n+1}+\left(\alpha_{n}-\alpha_{n-1}\right) z^{n}+\ldots \ldots \ldots+\left(\alpha_{1}-\alpha_{0}\right) z+\alpha_{0} \\
& +i\left\{\left(\beta_{n}-\beta_{n-1}\right) z^{n}+\ldots \ldots \ldots+\left(\beta_{1}-\beta_{0}\right) z+\beta_{0}\right\} \text {. } \\
& =-a_{n} z^{n+1}-\left(k_{1}-1\right) \alpha_{n} z^{n}+\left(k_{1} \alpha_{n}-\alpha_{n-1}\right) z^{n}+\left(k_{1} \alpha_{n-1}-\alpha_{n-2}\right) z^{n-1}+\ldots \ldots . \\
& +\left(k_{1} \alpha_{\lambda+1}-\alpha_{\lambda}\right) z^{\lambda+1}+\left(k_{1} \alpha_{\lambda}-\alpha_{\lambda-1}\right) z^{\lambda}+\left(\alpha_{\lambda-1}-\alpha_{\lambda-2}\right) z^{\lambda-1}+\left(\alpha_{\lambda-2}-\alpha_{\lambda-3}\right) z^{\lambda-2}+\ldots \ldots . \\
& +\left(\alpha_{2}-\alpha_{1}\right) z^{2}+\left(\alpha_{1}-\rho_{1} \alpha_{0}\right) z+\left(\rho_{1}-1\right) \alpha_{0} z+\alpha_{0}-\left(k_{1}-1\right)\left(\alpha_{n-1} z^{n-1}+\ldots \ldots . .+\alpha_{\lambda+1} z^{\lambda+1}+\alpha_{\lambda} z^{\lambda}\right) \\
& +i\left\{-\left(k_{2}-1\right) \beta_{n} z^{n}+\left(k_{2} \beta_{n}-\beta_{n-1}\right) z^{n}+\left(k_{2} \beta_{n-1}-\beta_{n-2}\right) z^{n-1}+\ldots \ldots\right. \\
& +\left(k_{2} \beta_{\mu+1}-\beta_{\mu}\right) z^{\mu+1}+\left(k_{2} \beta_{\mu}-\beta_{\mu-1}\right) z^{\mu}+\left(\beta_{\mu-1}-\beta_{\mu-2}\right) z^{\mu-1} \\
& +\left(\beta_{\mu-2}-\beta_{\mu-3}\right) z^{\mu-2}+\ldots \ldots . .+\left(\beta_{2}-\beta_{1}\right) z^{2}+\left(\beta_{1}-\rho_{2} \beta_{0}\right) z+\left(\rho_{2}-1\right) \beta_{0} z+\beta_{0} \\
& \left.\left.-\left(k_{2}-1\right)\left(\beta_{n-1} z^{n-1}+\ldots \ldots . .+\beta_{\mu+1} z^{\mu+1}+\beta_{\mu} z^{\mu}\right)\right\}\right] \\
& =z^{n}\left[-a_{n} z-\left(k_{1}-1\right) \alpha_{n}-i\left(k_{2}-1\right) \beta_{n}+\left(k_{1} \alpha_{n}-\alpha_{n-1}\right)+\left(k_{1} \alpha_{n-1}-\alpha_{n-2}\right) \frac{1}{z}+\right. \\
& +\left(k_{1} \alpha_{\lambda+1}-\alpha_{\lambda}\right) \frac{1}{z^{n-\lambda-1}}+\left(k_{1} \alpha_{\lambda}-\alpha_{\lambda-1}\right) \frac{1}{z^{n-\lambda}}+\left(\alpha_{\lambda-1}-\alpha_{\lambda-2}\right) \frac{1}{z^{n-\lambda+1}} \\
& +\left(\alpha_{\lambda-2}-\alpha_{\lambda-3}\right) \frac{1}{z^{n-\lambda+2}}+\ldots \ldots . .+\left(\alpha_{2}-\alpha_{1}\right) \frac{1}{z^{n-2}}+\left(\alpha_{1}-\rho_{1} \alpha_{0}\right) \frac{1}{z^{n-1}} \\
& +\left(\rho_{1}-1\right) \alpha_{0} \frac{1}{z^{n-1}}+\alpha_{0} \frac{1}{z^{n}}-\left(k_{1}-1\right)\left(\alpha_{n-1} \frac{1}{z}+\ldots \ldots . .+\alpha_{\lambda} \frac{1}{z^{n-\lambda}}\right) \\
& +i\left\{\left(k_{2} \beta_{n}-\beta_{n-1}\right)+\left(k_{2} \beta_{n-1}-\beta_{n-2}\right) \frac{1}{z}+\ldots \ldots\right. \\
& +\left(k_{2} \beta_{\mu+1}-\beta_{\mu}\right) \frac{1}{z^{n-\mu-1}}+\left(k_{2} \beta_{\mu}-\beta_{\mu-1}\right) \frac{1}{z^{n-\mu}}+\left(\beta_{\mu-1}-\beta_{\mu-2}\right) \frac{1}{z^{n-\mu+1}} \\
& +\left(\beta_{\mu-2}-\beta_{\mu-3}\right) \frac{1}{z^{n-\mu+2}}+\ldots \ldots . .+\left(\beta_{2}-\beta_{1}\right) \frac{1}{z^{n-2}}+\left(\beta_{1}-\rho_{2} \beta_{0}\right) \frac{1}{z^{n-1}} \\
& \left.\left.+\left(\rho_{2}-1\right) \beta_{0} \frac{1}{z^{n-1}}+\frac{\beta_{0}}{z^{n}}-\left(k_{2}-1\right)\left(\beta_{n-1} \frac{1}{z}+\ldots \ldots . .+\beta_{\mu} \frac{1}{z^{n-\mu}}\right)\right\}\right]
\end{aligned}
$$

For $|z|>1$ so that $\frac{1}{|z|^{n-j}}<1, j=0,1,2, \ldots \ldots \ldots, n$.We have by using the hypothesis, 
M. H. Gulzar and A. W. Manzoor/ BIBECHANA 13 (2016) 1-8 : RCOST p.6 (Online Publication: Dec., 2015)

$$
\begin{aligned}
|F(z)| \geq & \left.z\right|^{n}\left[\left|a_{n} z+\left(k_{1}-1\right) \alpha_{n}+i\left(k_{2}-1\right) \beta_{n}\right|-\left\{\left|k_{1} \alpha_{n}-\alpha_{n-1}\right|+\left|k_{1} \alpha_{n-1}-\alpha_{n-2}\right| \frac{1}{|z|}\right.\right. \\
& +\left|k_{1} \alpha_{\lambda}-\alpha_{\lambda-1}\right| \frac{1}{|z|^{n-\lambda}}+\left|\alpha_{\lambda-1}-\alpha_{\lambda-2}\right| \frac{1}{|z|^{n-\lambda+1}}+\left|\alpha_{\lambda-2}-\alpha_{\lambda-3}\right| \frac{1}{|z|^{n-\lambda+2}}+\ldots \ldots . \\
& +\left|\alpha_{2}-\alpha_{1}\right| \frac{1}{|z|^{n-2}}+\left|\alpha_{1}-\rho_{1} \alpha_{0}\right| \frac{1}{|z|^{n-1}}+\left|\rho_{1}-1\right|\left|\alpha_{0}\right| \frac{1}{|z|^{n-1}}+\left|\alpha_{0}\right| \frac{1}{|z|^{n-1}} \\
& -\left(k_{1}-1\right)\left(\left|\alpha_{n-1}\right| \frac{1}{|z|}+\ldots \ldots . .+\left|\alpha_{\lambda}\right| \frac{1}{|z|^{n-\lambda}}\right)+\left|k_{2} \beta_{n}-\beta_{n-1}\right|+\left|k_{2} \beta_{n-1}-\beta_{n-2}\right| \frac{1}{|z|}+\ldots \ldots . . \\
& +\left|k_{2} \beta_{\mu+1}-\beta_{\mu}\right| \frac{1}{|z|^{n-\mu-1}}+\left|k_{2} \beta_{\mu}-\beta_{\mu-1}\right| \frac{1}{|z|^{n-\mu}}+\left|\beta_{\mu-1}-\beta_{\mu-2}\right| \frac{1}{|z|^{n-\mu+1}} \\
& +\left|\beta_{\mu-2}-\beta_{\mu-3}\right| \frac{1}{|z|^{n-\mu+2}}+\ldots \ldots . .+\left|\beta_{2}-\beta_{1}\right| \frac{1}{|z|^{n-2}}+\left|\beta_{1}-\rho_{2} \beta_{0}\right| \frac{1}{|z|^{n-1}} \\
& \left.\left.+\left|\rho_{2}-1\right|\left|\beta_{0}\right| \frac{1}{|z|^{n-1}}+\frac{\left|\beta_{0}\right|}{|z|^{n}}-\left(k_{2}-1\right)\left(\left|\beta_{n-1}\right| \frac{1}{|z|}+\ldots \ldots \ldots+\left|\beta_{\mu}\right| \frac{1}{|z|^{n-\mu}}\right)\right\}\right] \\
\geq & |z|^{n}\left[\left|a_{n} z+\left(k_{1}-1\right) \alpha_{n}+i\left(k_{2}-1\right) \beta_{n}\right|-\left\{k_{1} \alpha_{n}-\alpha_{n-1}+k_{1} \alpha_{n-1}-\alpha_{n-2}\right.\right. \\
& +k_{1} \alpha_{\lambda}-\alpha_{\lambda-1}+\alpha_{\lambda-1}-\alpha_{\lambda-2}+\alpha_{\lambda-2}-\alpha_{\lambda-3}+\ldots \ldots . . \\
& +\alpha_{1}-\rho_{1} \alpha_{0}+\left(1-\rho_{1}\right)\left|\alpha_{0}\right|+\left|\alpha_{0}\right| \\
& +\left(k_{1}-1\right)\left(\left|\alpha_{n-1}\right|+\ldots \ldots . .+\left|\alpha_{\lambda}\right|\right)+k_{2} \beta_{n}-\beta_{n-1}+k_{2} \beta_{n-1}-\beta_{n-2}+\ldots \ldots . . \\
& +k_{2} \beta_{\mu+1}-\beta_{\mu}+k_{2} \beta_{\mu}-\beta_{\mu-1}+\beta_{\mu-1}-\beta_{\mu-2} \\
& +\beta_{\mu-2}-\beta_{\mu-3}+\ldots \ldots . .+\beta_{2}-\beta_{1}+\beta_{1}-\rho_{2} \beta_{0} \\
& \left.\left.+\left(1-\rho_{2}\right)\left|\beta_{0}\right|+\left|\beta_{0}\right|-\left(k_{2}-1\right)\left(\left|\beta_{n-1}\right|+\ldots \ldots . .+\left|\beta_{\mu}\right|\right)\right\}\right]
\end{aligned}
$$$$
=|z|^{n}\left[\left|a_{n} z+\left(k_{1}-1\right) \alpha_{n}+i\left(k_{2}-1\right) \beta_{n}\right|-\left\{\left(\alpha_{n}+\beta_{n}\right)-\left(\rho_{1} \alpha_{0}+\rho_{2} \beta_{0}\right)+\left(1-\rho_{1}\right)\left|\alpha_{0}\right|+\left(1-\rho_{2}\right)\left|\beta_{0}\right|\right.\right.
$$$$
\left.+\left|\alpha_{0}\right|+\left|\beta_{0}\right|+\left(k_{1}-1\right)\left(\sum_{j=\lambda}^{n}\left(\alpha_{j}+\left|\alpha_{j}\right|\right)\right)-\left(k_{1}-1\right)\left|\alpha_{n}\right|+\left(k_{2}-1\right)\left(\sum_{j=\mu}^{n}\left(\beta_{j}+\left|\beta_{j}\right|\right)-\left(k_{2}-1\right)\left|\beta_{n}\right|\right\}\right]
$$$$
>0
$$

if

$$
\begin{aligned}
\left|a_{n} z+\left(k_{1}-1\right) \alpha_{n}+i\left(k_{2}-1\right) \beta_{n}\right| & >\left(\alpha_{n}+\beta_{n}\right)-\left(\rho_{1} \alpha_{0}+\rho_{2} \beta_{0}\right)+\left(1-\rho_{1}\right)\left|\alpha_{0}\right|+\left(1-\rho_{2}\right)\left|\beta_{0}\right| \\
& +\left|\alpha_{0}\right|+\left|\beta_{0}\right|+\left(k_{1}-1\right)\left(\sum_{j=\lambda}^{n}\left(\alpha_{j}+\left|\alpha_{j}\right|\right)-\left(k_{1}-1\right)\left|\alpha_{n}\right|\right. \\
& +\left(k_{2}-1\right)\left(\sum_{j=\mu}^{n}\left(\beta_{j}+\left|\beta_{j}\right|\right)-\left(k_{2}-1\right)\left|\beta_{n}\right|\right.
\end{aligned}
$$

that is, if 
M. H. Gulzar and A. W. Manzoor/ BIBECHANA 13 (2016) 1-8 : RCOST p.7 (Online Publication: Dec., 2015)

$$
\begin{aligned}
& \left|z+\frac{\left(k_{1}-1\right) \alpha_{n}+i\left(k_{2}-1\right) \beta_{n}}{a_{n}}\right|>\frac{1}{\left|a_{n}\right|}\left[\left(\alpha_{n}+\beta_{n}\right)-\left(\rho_{1} \alpha_{0}+\rho_{2} \beta_{0}\right)+\left(1-\rho_{1}\right)\left|\alpha_{0}\right|+\left(1-\rho_{2}\right)\left|\beta_{0}\right|+\left(\left|\alpha_{0}\right|+\left|\beta_{0}\right|\right)\right. \\
& +(k-1)\left(\sum_{j=\lambda}^{n}\left(\alpha_{j}+\mid \alpha_{j}\right)\right)-\left(k_{1}-1\right)\left|\alpha_{n}\right| \\
& +\left(k_{2}-1\right)\left(\sum_{j=\lambda}^{n}\left(\beta_{j}+\left|\beta_{j}\right|\right)-\left(k_{2}-1\right)\left|\beta_{n}\right|\right.
\end{aligned}
$$

This shows that those zeros of $\mathrm{F}(\mathrm{z})$ whose modulus is greater than 1 lie in

$$
\begin{aligned}
\left|z+\frac{\left(k_{1}-1\right) \alpha_{n}+i\left(k_{2}-1\right) \beta_{n}}{a_{n}}\right| & \leq \frac{1}{\left|a_{n}\right|}\left[\left(\alpha_{n}+\beta_{n}\right)-\left(\rho_{1} \alpha_{0}+\rho_{2} \beta_{0}\right)+\left(1-\rho_{1}\right)\left|\alpha_{0}\right|+\left(1-\rho_{2}\right)\left|\beta_{0}\right|+\left(\left|\alpha_{0}\right|+\left|\beta_{0}\right|\right)\right. \\
& +(k-1)\left(\sum_{j=\lambda}^{n}\left(\alpha_{j}+\left|\alpha_{j}\right|\right)\right)-\left(k_{1}-1\right)\left|\alpha_{n}\right| \\
& +\left(k_{2}-1\right)\left(\sum_{j=\lambda}^{n}\left(\beta_{j}+\left|\beta_{j}\right|\right)-\left(k_{2}-1\right)\left|\beta_{n}\right|\right]
\end{aligned}
$$

Since those zeros of $F(z)$ whose modulus is less than or equal to 1 already satisfy the above inequality, it follows that all the zeros of $F(z)$ and hence of $p(z)$ lie in

$$
\begin{aligned}
\left|z+\frac{\left(k_{1}-1\right) \alpha_{n}+i\left(k_{2}-1\right) \beta_{n} \mid}{a_{n}}\right| & \leq \frac{1}{\left|a_{n}\right|}\left[\left(\alpha_{n}+\beta_{n}\right)-\left(\rho_{1} \alpha_{0}+\rho_{2} \beta_{0}\right)+\left(1-\rho_{1}\right)\left|\alpha_{0}\right|+\left(1-\rho_{2}\right)\left|\beta_{0}\right|+\left(\left|\alpha_{0}\right|+\left|\beta_{0}\right|\right)\right. \\
& +(k-1)\left(\sum_{j=\lambda}^{n}\left(\alpha_{j}+\left|\alpha_{j}\right|\right)\right)-\left(k_{1}-1\right)\left|\alpha_{n}\right| \\
& +\left(k_{2}-1\right)\left(\sum_{j=\lambda}^{n}\left(\beta_{j}+\left|\beta_{j}\right|\right)-\left(k_{2}-1\right)\left|\beta_{n}\right|\right]
\end{aligned}
$$

This completes the proof of Theorem 1.

\section{References}

[1] M. Marden, Geometry of Polynomials, Math. Surveys No.3, Amer. Math. Soc. Providence R.I. 1996.

[2] G.V. Milovanovic, D.S. Mitrinovic and T.M. Rassias, Topics in Polynomials, Extremal Problems, Inequalities, Zeros. World Scientific Publishing Co. River Edge, NJ, 1994.

[3] Q. I. Rahman and G. Schmeisser, Analytic Theory of polynomials, Oxford University Press, 2002.

[4] A. Joyal, G. Labelle and Q.I. Rahman, Canad. Math. Bull. 10 (1967) 53-63.

[5] A. Aziz and B. A. Zargar, Mathematiki, 31(1996) 239-244. 
M. H. Gulzar and A. W. Manzoor/ BIBECHANA 13 (2016) 1-8 : RCOST p.8 (Online Publication: Dec., 2015)

[6] W. M. Shah and A. Liman, On Enestrom-kakeya Theorem and related Analytic Functions, Proc. Indian Acad. Sci (Math Sci) 17 ( 3) (2007)359-370.

[7] A. Liman, Tawheeda Rasool and W.M. Shah, Bibechana, 10(2014) 71-81.

[8] Gulshan Singh, American Journal of Mathematical Analysis, 2 (1) (2014) 15- 18.

[9] M. H. Gulzar, International Journal of Innovative Research in Engineering and Science, 4( 3 )(2014) 33-36. 